\title{
RABIES ENCEPHALITIS WITH AN UNUSUALLY LONG LATENCY PERIOD
}

\author{
Umaamaheshwari Rajeswari Subramanian'1, Sundaramurthy Ganesan², Karthigeyan Thanjavur Sethuraman ${ }^{3}$, Ramesh Baskaran $^{4}$
}

1Final Year Postgraduate, Department of Internal Medicine, Madras Medical College, Chennai.

2 Professor, Department of Internal Medicine, Madras Medical College, Chennai.

${ }^{3}$ Assistant Professor, Department of Internal Medicine, Madras Medical College, Chennai.

${ }_{4}^{4}$ Assistant Professor, Department of Internal Medicine, Madras Medical College, Chennai.

\section{ABSTRACT}

\section{BACKGROUND}

Rabies is a preventable viral disease. In India, rabies is transmitted to humans mainly by dogs. Rabies has an incubation period lasting for several days to months or years. Here, we report a case of a young male who presented with aerophobia and hydrophobia, about twenty years after a suspected rabid dog bite, thus suggesting that rabies virus may have an unusually long incubation period.

\section{KEYWORDS}

Rabies, Encephalitis, Incubation Period, RT-PCR.

HOW TO CITE THIS ARTICLE: Subramanian UR, Ganesan S, Sethuraman KT, et al. Rabies encephalitis with an unusually long latency period. J. Evolution Med. Dent. Sci. 2016;5(93):6904-6905, DOI: 10.14260/Jemds/2016/1562

\section{BACKGROUND}

Rabies is a preventable viral disease transmitted by infected (rabid) animal to humans. In India, rabies is transmitted to humans, mainly by dogs. Other wild animals that are known to transmit rabies include bats, raccoons, skunks and foxes. According to the World Health Organisation data, the incidence of deaths due to rabies is much higher in India than other countries. ${ }^{1}$ In India about 3.5 million dog bite cases are registered every year. But the number of deaths in India has started declining from 30,000 in 1985 to 21,404 in 2004. Rabies has an incubation period lasting for several days to months or years. Here, we report a case of rabies with an unusually long incubation period.

\section{CASE REPORT}

A 26-year-old male patient was admitted with history of breathing difficulty and abnormal twitching of muscles over the trunk region for one day. On examination, the patient was awake and responding to simple commands. Patient was noted to have hydrophobia and aerophobia. The patient's mother gave a history of dog bite on his right hand twenty years back, which had resulted in a deep wound with bleeding. It was not known whether the dog had rabid symptoms or not.

A course of vaccination (five doses of ARV) was given in a local hospital. There was no history suggestive of Rabies immunoglobulin administration at the wound site at that time. The wound was not sutured and it had healed over the next few days. There was no subsequent known dog bite, cat bite, rat bite or any other wild animal bite.

On the day of admission, the patient had aerophobia, hydrophobia and drooling of saliva, all of which worsened progressively. Neurophysician's opinion was obtained.

Financial or Other, Competing Interest: None.

Submission 15-10-2016, Peer Review 08-11-2016,

Acceptance 14-11-2016, Published 21-11-2016.

Corresponding Author:

Dr. Umaamaheshwari Rajeswari Subramanian,

Final Year Postgraduate,

Department of Internal Medicine,

Madras Medical College-

Rajiv Gandhi Government General Hospital,

Chennai-600003.

E-mail:drumamakeswari@gmail.com

DOI: $10.14260 /$ jemds $/ 2016 / 1562$

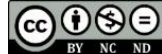

Based on the clinical picture and background history of dog bite 20 years back, a provisional diagnosis of rabies encephalitis was made.

Patient was isolated and treated with intravenous fluids, sedatives and supportive measures. After ruling out papilloedema, a lumbar puncture was done. Cerebrospinal fluid was sent for RT-PCR (rabies) to Government Veterinary College Hospital, Vepery, Chennai.

Despite the supportive care, patient's condition deteriorated and he developed cardiorespiratory arrest. Resuscitation was unsuccessful. An autopsy was advised, but the patient's attenders were not willing for it.

The cerebrospinal fluid report came as "RT-PCR" positive for rabies virus.

This case indicates that there is a possibility of long-term latency of the rabies virus.

\section{DISCUSSION}

Rabies is a rapidly progressive, acute infectious disease of the Central Nervous System (CNS) in humans and animals caused by the rabies virus. The infection is usually transmitted from animal vectors. ${ }^{2}$ Rabies virus is a lyssavirus belonging to the family Rhabdoviridae. ${ }^{1}$ It infects a broad range of animals and causes serious neurologic disease when transmitted to humans. It is a single-stranded RNA virus.

\section{There are three Modes of Transmission for the Rabies Infection}

1. Through animal bites.

2. Non-bite exposure to the saliva of the infected animal.

3. Human-to-human transmission.

In India, dogs constitute the most important source of rabies infection.

The incubation period of rabies (defined as the interval between exposure and the onset of clinical disease) is usually 20 to 90 days. ${ }^{2}$ But in rare cases, the incubation period has been documented to be extremely short (few days) or unusually long (few years). Initially, the rabies virus remains near the site of inoculation. Then it spreads centripetally along the peripheral nerves towards the central nervous system at a rate of up to $250 \mathrm{~mm}$ /day via retrograde fast axonal transport to the spinal cord or brainstem. ${ }^{2}$ Once the virus enters the CNS, 
it gets disseminated and spreads centrifugally to skin, salivary glands and autonomic nervous system.

The clinical picture includes a prodromal stage followed by severe neurological manifestations in the form of undue salivation, aerophobia and hydrophobia (furious rabies) or paralysis (dumb rabies). The very first clinical symptom is neuropathic pain or tingling sensation at the site of infection. ${ }^{1}$ Then there may be prodromal symptoms like fever and malaise followed by the neurological symptoms. Rabies encephalitis is distinguished by early brainstem involvement, which results in the classic features of hydrophobia and aerophobia. ${ }^{2}$ These symptoms are probably due to dysfunction of infected brainstem neurons that normally inhibit inspiratory neurons near the Nucleus Ambiguus. ${ }^{2}$ Hence, the defensive reflexes that protect the respiratory tract get exaggerated, resulting in aerophobia and hydrophobia. Brainstem dysfunction progresses rapidly and coma ensues. Usually, the patient dies within a few days.

Most of the rabies cases have an incubation period in the range of 31 to 90 days. In the literature, the overall reported incubation period varies from 30 days in $30 \%$ of the cases, 31 - 90 days in $54 \%$ to greater than 90 days in $15 \% .^{3}$ In $1 \%$ of the cases, an incubation period of more than one year has been recorded. In a cohort of 47 cases at a single centre in South India, the median incubation period recorded was 60 days (range of 7 days to 4 years). A case of rabies in a Vietnamese girl who immigrated to Australia was reported with the incubation period exceeding 6.5 years. ${ }^{4}$ There is one documented case report of Rabies Encephalitis after 25 years of latency period. ${ }^{3}$
In our case, the patient developed a rabies encephalitis twenty years after a dog bite.

\section{CONCLUSION}

This case emphasises that rabies can have an unusually long latency period (20 years). The patient had received ARV vaccine, but had not received any immunoglobulins. The need for vaccination along with immunoglobulins in category 3 bites is thus essential. The diagnosis of rabies encephalitis should be borne in mind in any patient presenting with features of acute encephalitis, especially so in cases with past history of animal bite (irrespective of the duration of latency period).

\section{REFERENCES}

1. Yousaf MZ, Qasim M, Zia S, et al. Rabies molecular virology, diagnosis, prevention and treatment. Virol Journal 2012;9:50.

2. Jackson AC. Harrison's principles of internal medicine. Chapter 232. New York: McGraw-Hill Education 2015: P. 1299.

3. Shankar SK, Mahadevan A, Sapico SD, et al. Rabies viral encephalitis with proable 25 year incubation period! Ann Indian Acad Neurol 2012;15(3):221-3.

4. Johnson N, Fooks A, McColl K. Re-examination of human rabies case with long incubation, Australia. Emerging Infectious Diseases 2008;14(12):1950-51. www.cdc.gov/eid 